7th International Workshop on Astronomy and

Relativistic Astrophysics (IWARA 2016)

International Journal of Modern Physics: Conference Series

Vol. 45 (2017) 1760013 (6 pages)

(C) The Author(s)

DOI: $10.1142 / \mathrm{S} 2010194517600138$

\title{
STEP as a Decisive Test of MOND on Earth
}

\author{
Jonas P. Pereira \\ Centro de Ciências Naturais e Humanas \\ Universidade Federal do $A B C$ \\ Avenida dos Estados 5001, CEP 09210-580, Santo André, SP, Brazil \\ jonas.pereira@ufabc.edu.br
}

Published 15 August 2017

\begin{abstract}
We review and emphasize the importance of the Satellite Test of the Equivalence Principle (STEP) in probing one of the most successful and popular alternative theories to dark matter known as Modified Newtonian Dynamics (MOND), on Earth. This would be achieved with no modification of STEP's current design and sensitivity and if MOND exists STEP could in principle easily detect it.
\end{abstract}

Keywords: MOND, STEP, Dark Matter.

PACS numbers: 04.50

\section{Introduction}

Modified Newtonian Dynamics, or MOND for short, was proposed by Milgrom in the beginning of the 1980's in order to explain the flat aspect rotation curves of galaxies exhibited (asymptotic constancy of test-particles' orbital velocities, more easily observed in spiral galaxies). ${ }^{1-3}$ It was meant to replace the dark matter's paradigm by assuming that Newton's second law should be replaced by

$$
\vec{F}=m_{i} \mu\left(\frac{|\vec{a}|}{a_{0}}\right) \vec{a},
$$

where $m_{i}$ is the particle's inertial mass, $\vec{a}$ its acceleration and $\mu(x), x \doteq|\vec{a}| / a_{0}$, is the so-called interpolating function, which introduces the acceleration scale $a_{0} \simeq$ $10^{-10} \mathrm{~ms}^{-2}$. (This value is obtained when MOND's asymptotic orbital velocities, $V_{\infty}^{4}=M G a_{0}, M$ the baryonic [luminous] matter of the galaxy, agrees with all observed values of $V_{\infty}$ and $M$ for galaxies.) In order to recover Newton's second

This is an Open Access article published by World Scientific Publishing Company. It is distributed under the terms of the Creative Commons Attribution 4.0 (CC-BY) License. Further distribution of this work is permitted, provided the original work is properly cited. 
law for large accelerations and constant orbital velocities in the regime of small accelerations, $\mu$ should satisfy the constraints

$$
\mu(x)=\left\{\begin{array}{l}
1, x \gg 1 \\
x, x \ll 1
\end{array}\right.
$$

It is important to stress that so far there is not yet an unequivocally (observationally) established interpolating function fulfilling the above equation.

In Ref. ${ }^{4}$ we have proposed a simple way to test MOND on Earth by means of the experiment-to-be called the Satellite Test of the Equivalence Principle (STEP), without any change of its current aspects. Its importance lies on the fact that to date there are no Earth-based scalar experiments of MOND, quite oppositely to dark matter (DM) particle experiments, such as PandaX (see Ref. ${ }^{5}$ ) and LUX , ${ }^{6}$ though nothing has yet been found. We stress, though, that there already exist Earth-based experimental proposals to MOND and they were pioneered by Ignatiev not long ago.$^{7}$ An enhancement of Ignatiev's idea has already been put forward.$^{8}$ Nevertheless, in all of these suggestions vectorial quantities are involved, such as accelerations, and their control to the precision required by MOND still seems challenging. For instance, MOND experiments à la Ignatiev would need the knowledge and the control of the resultant acceleration on Earth's spots (due to Earth itself, Earth's orbit around the Sun, Sun's rotation around the center of mass of our galaxy, among others) with a precision of at least ten digits. This may be difficult due to the fact even the presence of an experimentalist some meters from the experiment could induce accelerations larger than MOND's scale. Therefore, it seems more promising to work with proposals with less degrees of freedom, for instance dealing with scalar observables and test-particles constrained to move along given directions. STEP as MOND's test seems perfect in this sense, as we explain in the sequel.

STEP was put forward by Stanford University in the early 1970's (see Ref. ${ }^{9}$ ) and has been enhanced since then,${ }^{10,11}$ though it has not yet been launched. It was conceived with the purpose of testing the Weak Equivalence Principle (WEP), vital for the geometric interpretation of general relativity (GR).$^{12}$ It consists of a generalization of Galileo's free fall experiments in which a satellite carrying two pairs of test particles of different natures will orbit around Earth for six months at an altitude of approximately $500 \mathrm{Km}$ (see Refs. ${ }^{10,11}$ ). The motions and accelerations of these test-particles will be monitored with the help of very sensitive superconducting devices known as SQUIDs (see Refs. ${ }^{10,11}$ ). SQUID devices are such that they induce reactive forces on the test-particles, leading them to behave as harmonic oscillators with periods around $1000 \mathrm{~s}$ for STEP's fiducial parameters.$^{9-11}$ They can measure both test-particles' common and differential accelerations with a current precision of $10^{-19} \mathrm{~g}$ and $10^{-18} \mathrm{~g}$, respectively ${ }^{10,11}$ where $\mathrm{g}$ is Earth's surface gravity. Nevertheless, position sensitivities are poor and are currently estimated to be around $10^{2} \mathrm{fm}$. The reason for large acceleration precision and poor position sensitivity is due to an intrinsic trade-off between these quantities, inherent in SQUID devices, ${ }^{9}$ 
and this is in clear consonance with the WEP's test, which is naturally revealed by means of non-null acceleration differences between two freely-falling particles.

STEP is endowed with thrusters that can cancel out dragging forces present on the spacecraft, due for instance to Earth's atmosphere and light rays, allowing thus the satellite to "float" around Earth (drag-free mode ${ }^{10}$ ). In this situation testparticles would have very small amplitudes of oscillation with respect to the walls of STEP, around $10^{-10}-10^{-8} \mathrm{~m}$. For the case the system is not in drag-free operation higher amplitudes may be reached, up to the hard limit of some millimeters ${ }^{9}$

As clear from the above, opportunity presents itself to probe MOND with STEP because it naturally induces test-particles' motions and serendipitously enough it is always possible to find scenarios where local accelerations are much smaller than $a_{0}$. This is the case for instance during the drag-free mode, where accelerations on test-particles should be no larger than $10^{-14} \mathrm{~g}$. Nevertheless, since the effective (reactive) forces acting on the test particles are not of gravitational nature, MOND would be tested with STEP as modified inertia. Almost nothing is known in this case,${ }^{13}$ though there are already evidences ${ }^{13}$ it should present aspects quite different from MOND as modified gravity (currently adopted in all astrophysical and cosmological analyses). ${ }^{14}$ This means that in order to come up with predictions and target observables, one should not disregard any possible scenario MOND as modified inertia could have within STEP. In particular, one should pay careful attention to the Strong Equivalence Principle (SEP), ${ }^{12}$ as it is violated in MOND as modified gravity ${ }^{14}$ Now we summarize all possible scenarios MOND and STEP could present.

\section{MOND When the SEP Holds}

\subsection{Drag-free operation}

When the SEP is valid one can always insert locally inertial frames where Milgrom's law of motion holds. When the system is in drag-free mode, accelerations are much smaller than $a_{0}$ and the axes could always be oriented such that the equation of motion of a given test-particle is $(z>0)$ (see Ref. ${ }^{4}$ )

$$
\ddot{\bar{z}}+\sqrt{\frac{a_{0}}{z_{0}}} \omega \sqrt{\bar{z}}=0,
$$

where $\bar{z} \doteq z / z_{0}, z_{0}$ is a constant representing the amplitude of motion, and $\omega$ is the frequency of the oscillations. Generic aspects of the effective restoring force naturally imply the motion is oscillatory, but from Eq. (3) it clearly is not harmonic. The aforesaid equation can be solved analytically in terms of hypergeometric functions. However, we present here its numerical solutions by taking as the initial condition it is released from rest at $\bar{z}=1$. Figure 1 shows the outcome of such case for different amplitudes. One notices that departures from Newtonian dynamics are very easy to be noticed with regard to the periods of oscillation of the test-masses $\left(4 t_{0}\right.$, where 


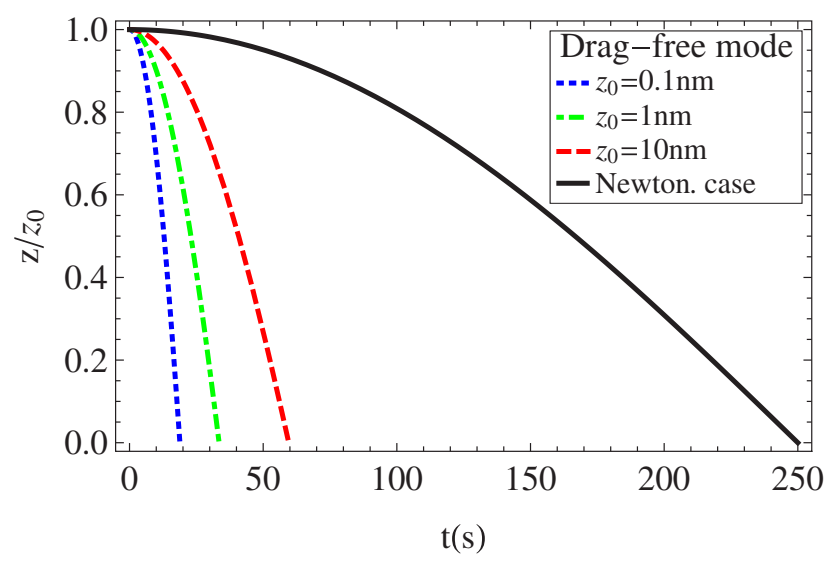

Fig. 1. Test particle's dynamics for some $z_{0}$ such that $a \ll a_{0}\left(\mu\left(a / a_{0}\right) \simeq a / a_{0}\right)$. Frequencies are very easy-to-measure observables in this case.$^{4}$

$t_{0}$ is defined by $z\left(t_{0}\right)=0$ ), as one already expects due to the fact the test-particles are putatively in deep MOND regime.

\subsection{Non-Drag-Free Operation}

Another possibility that arises in the case the SEP holds is when the system is not in the drag-free mode. In this case large amplitudes of oscillation are expected, which implies accelerations larger than $a_{0}$. For making predictions here one has to make use of specific interpolating functions $\mu$. In the context of MOND as modified inertia, there is no assurance aspects valid in modified gravity MOND still hold true. Only data analysis will settle this point. Nevertheless, one could use some well-known astrophysically suggested interpolating functions as reference functions to data analysis. In this case, two thereof are of importance. The first one is the so-called "standard interpolation function", $\mu_{2}$, defined as

$$
\mu_{2}=\frac{x}{\sqrt{1+x^{2}}}
$$

a particular member of the $n$-family of interpolating functions $\mu_{n}=x / \sqrt[n]{1+x^{n}}$. MOND's equation of motion in this case, upon a convenient orientation of the axes, is cast as 4

$$
\ddot{\bar{z}}+\frac{1}{\sqrt{2}} \sqrt{\bar{z}^{2} \omega^{4}+\bar{z} \omega^{2} \sqrt{\omega^{4} \bar{z}^{2}+\frac{4 a_{0}^{2}}{z_{0}^{2}}}}=0 .
$$

The second one is the naturally-occurring interpolating function in Bekenstein's TeVeS (See Ref. ${ }^{15}$ ), namely

$$
\mu_{T V S}(x) \doteq \frac{\sqrt{1+4 x}-1}{\sqrt{1+4 x}+1},
$$




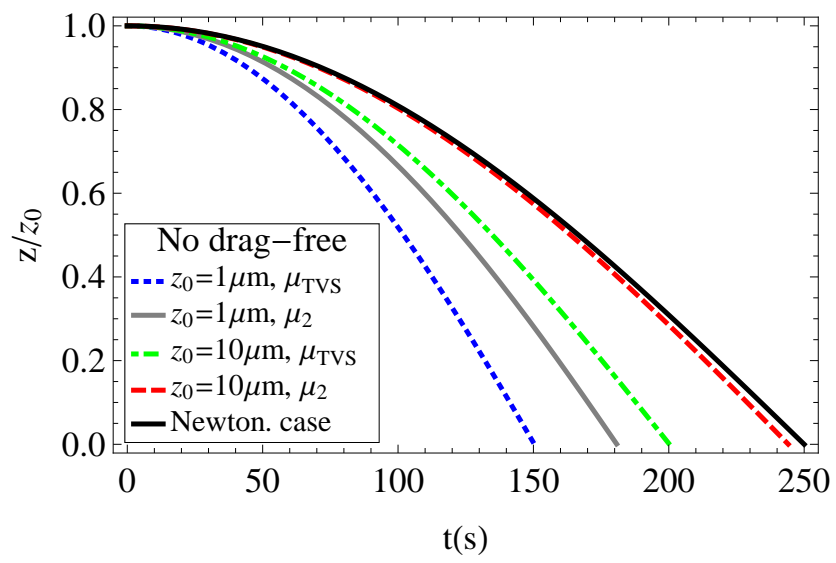

Fig. 2. Test particle's dynamics for some $z_{0}$ and $\mu_{2}$ and $\mu_{T V S} \cdot{ }^{4}$

whose associated equation of motion is 4

$$
\ddot{\bar{z}}+\omega^{2} \bar{z}+\sqrt{\frac{a_{0}}{z_{0}}} \omega \sqrt{\bar{z}}=0 .
$$

Figure 2 exhibits the numerical integration of Eqs. (5) and (7) for some selected amplitudes. Note that solutions involving $\mu_{2}$ go quicker to their classical counterparts than $\mu_{T V S}$ do. Nevertheless, it can be shown ${ }^{4}$ that even near the hard limit of amplitudes cumulative effects could always evidence the difference between the dynamics. For instance, after a week of STEP's operation, only by counting the number of times test-particles hit their turning points would make it possible to differ Newton's from Milgrom's dynamics.

\section{MOND When the SEP is Violated}

The last possible scenario for MOND in the context of STEP is when the SEP does not hold. This case is beguiling because it would require MOND's formulation in non-inertial coordinate systems, not yet known. In order to come up with predictions and the realization of the physically interesting observables, one has to propose models. Here we put forward a simple one that assumes (i) the validity of the WEP and (ii) the validity of the vectorial sum of accelerations within MOND. These assumptions are reasonable given the already existent constraints on the universality of the free-fall due to torsion balances and the absolute nature of time in non-relativistic MOND. When assumptions (i) and (ii) are taken into account and compositions of reference frames are done, as well as the intrinsic statistical nature of STEP, one ends up with the following equation of motion for a test-particle in a local coordinate frame ${ }^{4}$

$$
\ddot{\bar{z}} \approx-\frac{\omega^{2} \bar{z}}{\mu(y)+\frac{y}{2} \frac{\partial \mu(y)}{\partial y}},
$$


where $y \approx g / a_{0} \approx 10^{11}$ for freely falling particles. In the above equation it is evident the influence of external quantities on the test-particle's internal dynamics, as expected when the SEP is violated. Given that $y \gg 1$, we have that the term in the denominator of Eq.(8) is very close to the unity. Nevertheless, it can be shown ${ }^{4}$ that due to cumulative effects several interpolating functions could be probed with STEP.

Even if the spacecraft is not launched, in principle MOND could still be probed with STEP. ${ }^{4}$ The reason for this is chiefly the possibility of allowing the system to run longer than when it is orbit, the possible enhancement of position sensitivities and the possibility of always eliminating Earth's gravity.

\section{Summary}

We have reviewed and stressed some of the main ideas of Ref. ${ }^{4}$ about the use of STEP in probing MOND as modified inertia and the Strong Equivalence Principle as well, on top of the Weak Equivalence Principle, without any modification of its planned design and sensitivity. Even if the spacecraft is not launched at all, there is still the possibility of probing MOND and the SEP. In summary, if deviations from Newton's second law are measured, then DM as it currently stands should be revisited, as well as GR. On the other hand, if no deviations are detected, then MOND should either be a particular change of gravity or not take place at all.

\section{Acknowledgments}

J. P. P. acknowledges the financial support given by Fundação de Amparo à Pesquisa do Estado de São Paulo (FAPESP) under the project No. 2015/04174-9.

\section{References}

1. M. Milgrom, Astrophys. J. 270, 365 (July 1983).

2. M. Milgrom, Astrophys. J. 270, 371 (July 1983).

3. M. Milgrom, Astrophys. J. 270, 384 (July 1983).

4. J. P. Pereira, J. M. Overduin, and A. J. Poyneer, Physical Review Letters 117, 071103 (August 2016).

5. A. Tan et al., Physical Review Letters 117, 121303 (September 2016).

6. D. S. Akerib et al., ArXiv e-prints (August 2016).

7. A. Y. Ignatiev, Physical Review Letters 98, 101101 (March 2007).

8. V. A. De Lorenci, M. Faúndez-Abans, and J. P. Pereira, A\&A 503, L1 (August 2009).

9. P. W. Worden, Jr., Acta Astronautica 5, 27 (February 1978).

10. J. Mester, R. Torii, P. Worden, N. Lockerbie, S. Vitale, and C. W. F. Everitt, Classical and Quantum Gravity 18, 2475 (July 2001).

11. J. Overduin, F. Everitt, P. Worden, and J. Mester, Classical and Quantum Gravity 29, 184012 (September 2012).

12. C. M. Will, Living Reviews in Relativity 17, 4 (June 2014).

13. M. Milgrom, Acta Phys. Pol. B 42, 2175 (2011).

14. J. Bekenstein and M. Milgrom, Astrophys. J. 286, 7 (November 1984).

15. J. D. Bekenstein, Physical Review D 70, 083509 (October 2004). 